\title{
ANÁLISE COMPARATIVA DA EFICIÊNCIA DE AGITAÇÃO EM TANQUES DE MISTURA COM DUPLO IMPELIDOR (ÂNCORA - PBT) EM REGIME LAMINAR ATRAVÉS DE CFD
}

\author{
A. C. CIDES $^{1}$, J.L. GOMEZ ${ }^{2}$ e E. CEKINSKI ${ }^{1}$ \\ ${ }^{1}$ Instituto de Pesquisas Tecnológicas, Departamento de Engenharia Química e de Alimentos. \\ ${ }^{2}$ Universidade Estadual de Campinas, Faculdade de Engenharia Química. \\ E-mail para contato: ac_paiva@hotmail.com
}

\begin{abstract}
RESUMO - A indústria caracteriza-se pela fabricação de produtos visando à otimização e qualidade de seus processos. Por isso é importante uma escolha adequada do sistema de agitação, a fim de evitar zonas estagnadas, não homogeneidade e aumento do tempo de processo. Neste trabalho se avalia e comparar um conjunto de dois impelidores com características de fluxo diferentes em um tanque de mistura através da modelagem CFD. O estudo consiste em comparar um sistema agitado padrão utilizando um impelidor âncora com um arranjo de duplo impelidor Âncora-PBT. Foram realizadas simulações para diversos fluidos viscosos operando em regime laminar utilizando um traçador numérico. Os resultados indicam um aumento na eficiência da agitação e redução do consumo de energia no arranjo proposto.
\end{abstract}

\section{INTRODUÇÃO}

As operações em tanques agitados são amplamente utilizadas e envolvem transformações físicas e químicas durante sua operação. Atualmente existe uma contínua busca na otimização dos processos através de melhorias nos equipamentos já existentes, visando à qualidade do produto, a capacidade e da eficiência dos equipamentos e redução de custo operacional. Durante a elaboração de projetos e dimensionamento dos equipamentos pode ser útil o uso de técnicas computacionais tais como CFD (Computational Fluid Dynamics) para ajudar a entender o fluxo dentro de um tanque de mistura (Versteeg e Malalasekera, 1995), sendo o software ANSYS-CFX um dos mais usados.

Cabe notar que o fluxo característico no tanque pode ser alterado por alguns parâmetros geométricos do impelidor, propriedades do fluido ou a utilização de diversos impelidores (Joaquim Jr. et al., 2007), portanto, durante a elaboração do projeto deverão ser analisadas as propriedades críticas dos produtos e do processo, afim de que o projeto do agitador e seus componentes contemplem todas as características dos produtos desejados.

No entanto, o estudo de sistemas de mistura apresenta diversas dificuldades e desafios que vem sendo pesquisados por diversos pesquisadores, porém o maior volume de estudos concentra-se no sistema composto por um impelidor por tanque. Estudos com dois impelidores não possuem ainda muitos trabalhos divulgados, principalmente relacionados a regime laminar. 
No estudo se avalia a influência de um segundo impelidor na otimização de um tanque de mistura já existente que possui um agitador tipo âncora. Foi contemplado um segundo impelidor tipo pás inclinadas (PBT) com características de fluxo diferente do impelidor existente. O objetivo é conseguir um aumento da eficiência da agitação e redução do tempo de processamento. Assim, foram realizadas análises da influência do clearance e da viscosidade utilizando o arranjo de duplo impelidor Âncora-PBT utilizando um traçador numérico. Finalmente, os resultados sobre o padrão de escoamento, potência e consumo de energia no tanque de mistura foram comparados com o sistema já existente e discutidos.

\section{MODELAGEM MATEMÁTICA}

O escoamento de um fluido dentro do tanque de mistura pode ser descrito por as equações de Navier-Stokes, as quais são obtidas a partir das leis de conservação de massa, momentum e energia. Estas equações permitem determinar os campos de velocidade e de pressão que agem no escoamento do fluido. A técnica CFD busca resolver estas equações de transporte que governa o sistema, discretizando o domínio em volumes elementares e satisfazendo as leis de conservação.

A seguir, serão apresentadas as equações que governa o movimento de um fluido em um sistema rotativo. Em que; $\rho, \vec{v}, \mu, P, \vec{g}$ são densidade, velocidade, viscosidade, pressão e gravidade respectivamente.

$$
\begin{aligned}
& \frac{\partial \rho}{\partial t}+\nabla \cdot(\rho \vec{v})=0 \\
& \rho\left[\frac{\partial v}{\partial t}+\nabla \cdot(v \cdot \vec{v})\right]=-\nabla p-\mu\left[\nabla \vec{v}+(\nabla \vec{v})^{T}-\frac{2}{3} \delta \nabla \cdot \vec{v}\right]+\rho \vec{g}+\sum S
\end{aligned}
$$

Entretanto, quando se considera um escoamento sob um sistema de referencia rotativo, deve-se acrescentar um termo fonte $S$ responsáveis pelas forças centrifuga e de Coriolis que aparecem no sistema (Ansys, 2011).

\section{IMPLEMENTAÇÃO CFD}

A geometria do tanque de mistura foi baseada nas dimensões de um tanque de escala industrial, consistente com um tanque cilíndrico de diâmetro $\mathrm{T}=2 \mathrm{~m}$ com fundo toroesférico 10\%. Foi estabelecido um primeiro arranjo com impelidor âncora e outro utilizando um sistema de duplo impelidor Âncora-PBT no tanque de mistura, conforme mostra a Figura 1. O segundo sistema é composto de um impelidor com quatro pás inclinadas a $45^{\circ}$, de forma descentralizada ao sistema já existente girando em sentido contrario.

Para o impelidor âncora foi utilizada um diâmetro $\mathrm{D}=0,96 \mathrm{~T}$ enquanto que o impelidor PBT foi de $\mathrm{D}=\mathrm{T} / 3$. Entretanto, as interfaces do domínio rotativo do impelidor PBT foram localizadas conforme sugestões feitas na literatura (Lane et al., 2000), no caso do impelidor âncora as interfases foram definidas pelo volume de revolução deste. 

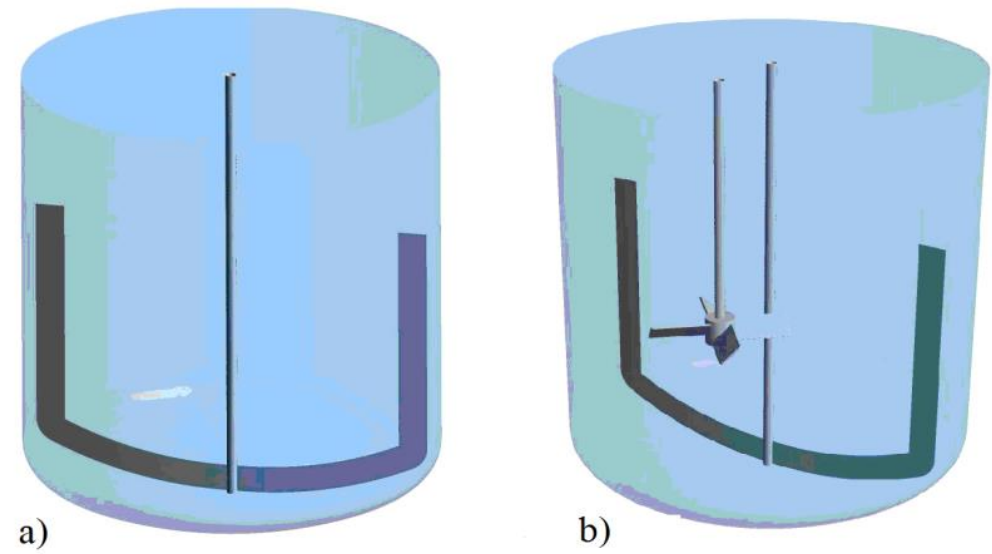

Figura 1 - Geometria do tanque e impelidor. a) Sistema Âncora. b) Sistema Âncora-PBT

Foi feita uma malha hibrida baseada principalmente em elementos tetraédricos e prismáticos, em que, realizou-se um teste de malha com diferentes refinamentos $(0.8,1.5$ e 2 milhões de elementos) principalmente nas paredes do tanque e proximidades dos impelidor, entretanto, foi empregada uma camada de elementos prismáticos em toda superfície do tanque e impelidor conforme visto na Figura 2.

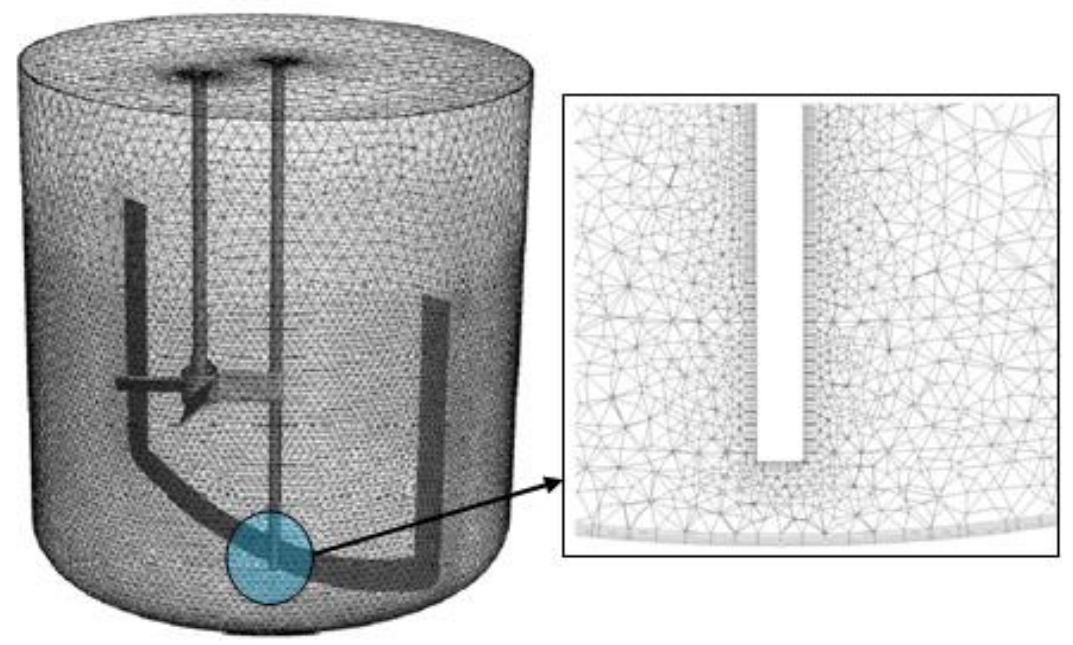

Figura 2 - Elementos prismáticos e tetraédricos. Sistema Âncora-PBT.

O efeito do número de elementos da malha sobre o cálculo das variáveis de interesse tais como potência e torque foram estudados. Finalmente, foi observado que acima de 2 milhões de elementos os resultados apresentam certa independência com variações inferiores a 3\% em concordância com critérios escolhidos por outros autores (Brucato et al. 1998).

Todas as simulações foram realizadas em estado estacionário para um escoamento monofásico incompressível, em regime laminar $(\operatorname{Re}<10)$. As condições de contorno de não escorregamento em todas as superfícies do tanque e impelidor, assim como a condição de 
superfície plana no topo do tanque foram empregadas. Uma abordagem de múltiplos sistemas de referencia (MFR-Frozen Rotor) na rotação impelidor e um esquema de discretização de alta ordem (High Resolution) também foram utilizados na configuração do modelo em CFD conforme sugestões feitas em trabalhos anteriores (Nunhez et al., 2012). Por ultimo, a fim de considerar a flexibilidade das operações de mistura um fluido com diferentes viscosidades $(25.000,50.000$ e $100.000 \mathrm{cP})$ foi criado.

\section{RESULTADOS E DISCUSSÕES}

Através de CFD se pretende predizer o escoamento no interior do tanque devido aos fluxos gerados pela movimentação dos impelidores, bem como a estimativa de parâmetros globais importantes no projeto de tanque. Além disso, contempla-se a utilização de um traçador numérico que permita estabelecer o tempo de mistura para os sistemas estudados.

Já que a técnica em CFD emprega um método numérico na resolução das equações de transporte foi escolhido um critério de convergência RMS (Root Mean Square) $<10^{-5}$ seguindo trabalhos da literatura (Murthy e Joshi, 2008), a fim de garantir que os erros residuais associados ao método não sejam significativos. A convergência da solução foi verificada através do monitoramento da estabilidade de algumas variáveis de interesse.

\subsection{Caracterização do Fluxo}

Sistema Âncora: Conforme descrito na literatura, o impelidor tipo âncora apresenta principalmente um padrão de escoamento tangencial visto na Figura 3. No entanto, os perfis indicam que o impelidor âncora possui pouco movimento axial.
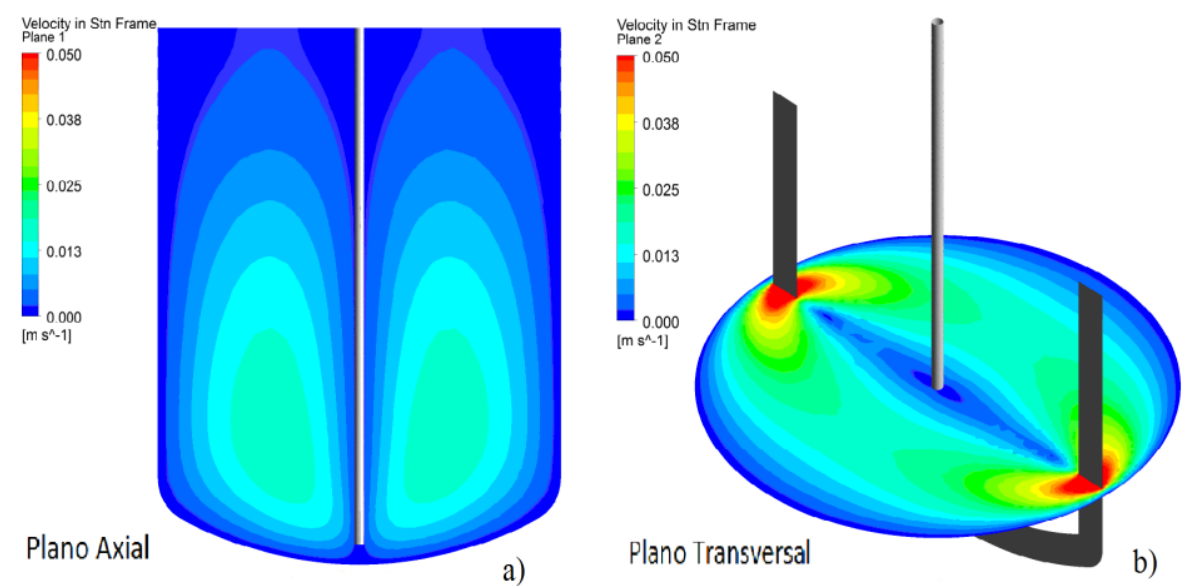

Figura 3 - Perfil de velocidade do impelidor âncora.

Sistema Âncora - PBT: Para se garantir uma maior homogeneidade pretende-se dimensionar um sistema para favorecer circulação radial e axial do escoamento no tanque. Com isso, sugere-se que um novo impelidor seja incluído ao sistema com o impelidor âncora para aumentar a recirculação do fluido no tanque de mistura. Para isso foi escolhido o impelidor de pás inclinadas a $45^{\circ}$, sendo um impelidor de fluxo mistos mais comuns e de baixo custo na indústria (Paul et al., 2004). 
Na Figura 4 os fluxos das velocidades estão expressos em contornos. Pode- se perceber que há deslocamento do fluido para o fundo do tanque, somando-se as parcelas axial e radial do impelidor PBT no perfil de velocidade do sistema. O impelidor PBT exerce sua função de gerar fluxo axial e radial, com isso, tende a impulsionar a massa líquida para o fundo do tanque.
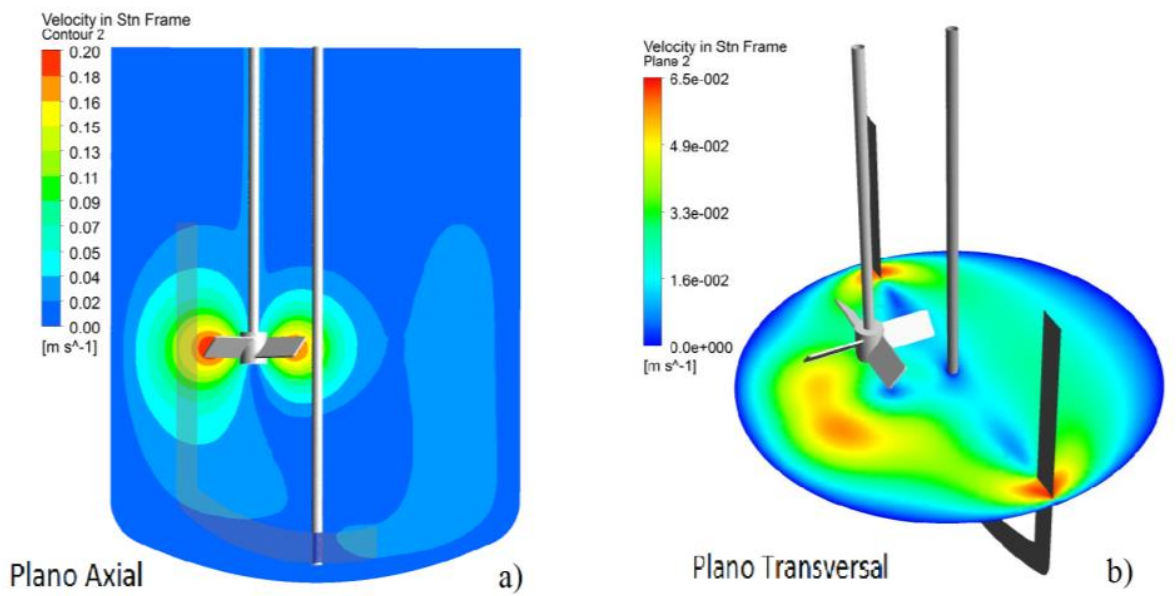

Figura 4 - Perfil de velocidade do conjunto de impelidos Âncora - PBT.

\subsection{Tempo de Mistura}

O tempo de mistura dentro de um tanque agitado é definido como o tempo medido do instante da adição de um traçador até o instante em que o líquido alcança um grau de uniformidade desejado (Joaquim Jr. et al., 2007). Na prática, o tempo de mistura pode ser medido adicionando um traçador até que o conteúdo do tanque atinja o grau de mistura especificado ( $95 \%$ ou $99 \%$ ) da concentração de equilíbrio $\left(\mathrm{C}^{\infty}\right)$. Este será dependente da forma em que é adicionado o traçador e a localização do detector (Chhabra e Richardson, 1999).

Frequentemente, para o cálculo do tempo de mistura utiliza-se um coeficiente de variância $\left(\sigma^{2}\right)$, o qual permite definir a variação da concentração do traçador $(C i)$ sobre o valor da concentração média a partir da amostragem em diferentes regiões do tanque $(\mathrm{N})$, descrito através da equação (4).

$$
\sigma^{2}=\frac{1}{N-1} \sum_{i=0}^{i=p}(C i-\bar{C})^{2}
$$

Em CFD, isso é feito utilizado um traçador numérico que represente uma concentração dentro do tanque. Assim, para determinar o tempo de mistura foi monitorado o coeficiente de variância do traçador como é observado na Figura 5, até atingir um critério de uniformidade desejado (Coroneo et al., 2010). 


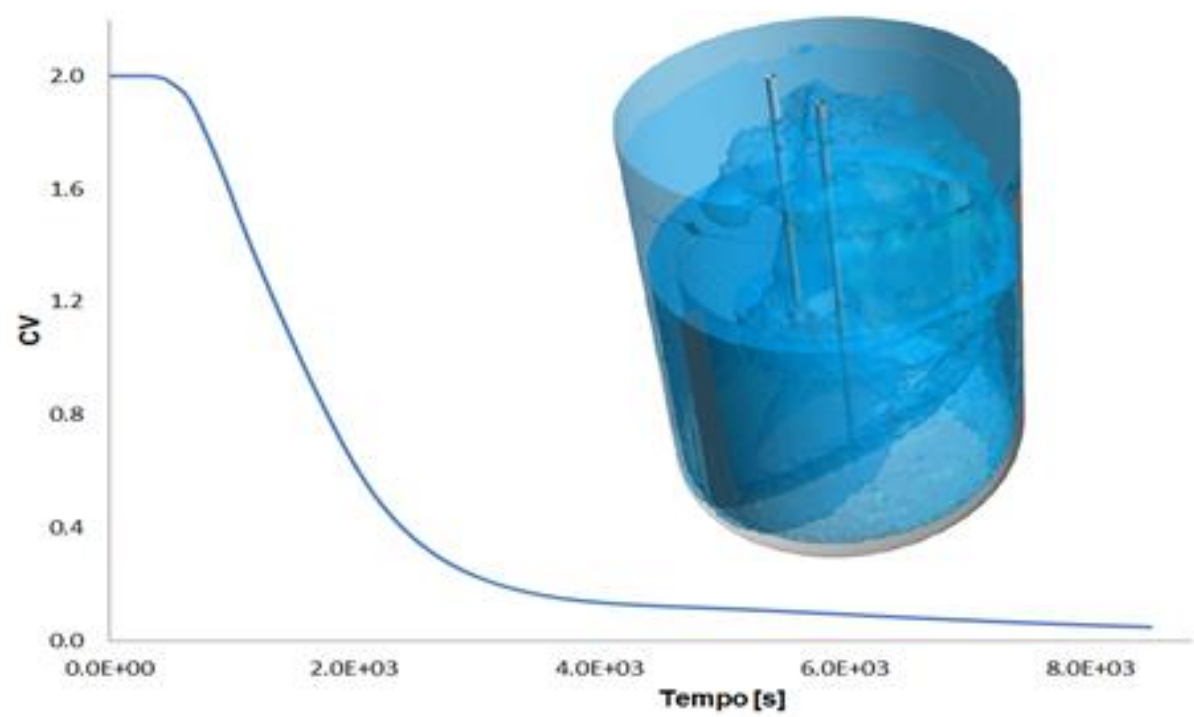

Figura 5 - Coeficiente de variância no decorrer do tempo. Sistema Âncora-PBT.

\subsection{Estimativa da potência e consumo de energia}

Para a estimativa da potência utilizou- se o valor do torque no impelidor calculado através das sub-rotinas estabelecidas em ANSYS 14.0. A potência total consumida no processo é definida como a soma da potência consumida por cada impelidor. O consumo de energia é determinado multiplicando-se a potência total pelo tempo de mistura, que corresponde ao tempo que o equipamento permanecerá em funcionamento. Entretanto, para facilitar a comparação dos resultados foi calculada uma potência especifica P/V (volume do tanque de 7,1 m3), apresentado na Tabela 1.

Tabela 1 - Comparação do consumo de energia os sistemas de estudo.

\begin{tabular}{|c|c|c|c|}
\hline Sistema & $\mathrm{P} / \mathrm{V}\left[\mathrm{W} \mathrm{m}^{\wedge}-3\right]$ & $\mathrm{t}$ mistura $[\mathrm{h}]$ & Energia $[\mathrm{KWh}]$ \\
\hline Âncora & 5,3 & 82,00 & 3,09 \\
\hline Âncora + PBT & 28,1 & 0,98 & 0,20 \\
\hline
\end{tabular}

Embora o sistema Âncora - PBT apresente a maior potência, o tempo requerido para atingir a mistura desejada é muito menor comparado com o sistema Âncora, portanto em termos de consumo de energia, a configuração impelidor/tanque utilizando duplo impelidor apresenta menor consumo energético.

Em busca de um melhoramento do sistema proposto de duplo impelidor Âncora - PBT foi realizado um estudo da influência do clearance do impelidor PBT sobre os fluxos gerados no tanque de mistura. Desse modo, foram feitas simulações com diversos clearance conforme reportadas na Tabela 2. 
Tabela 2 - Influencia da localização do impelidor PBT no consumo de energia

\begin{tabular}{|c|c|c|c|}
\hline Clearance & $\mathrm{P} / \mathrm{V}\left[\mathrm{W} \mathrm{m}^{\wedge}-3\right]$ & $\mathrm{t}$ mistura $[\mathrm{h}]$ & Energia [KWh] \\
\hline $1 / 2$ & 28,164 & 1,11 & 0,22 \\
\hline $1 / 3$ & 28,149 & 0,98 & 0,20 \\
\hline $1 / 4$ & 28,154 & 2,36 & 0,47 \\
\hline
\end{tabular}

Conforme a tabela acima se observa que mesmo variando o clearance, a potência requerida pelo sistema é quase a mesma, no entanto, o tempo de mistura é influenciado pela localização do impelidor. De acordo com os resultados, o clearence de 1/3 proporcionará um menor consumo energético pelo qual será a configuração a preferir. Esta análise pode ser comparada a literatura com o fato de que impelidores radiais colocados próximos ao fundo do tanque podem ser eficientes, mas pode haver problemas na uniformidade da mistura.

Por outro lado, devido á necessidade atual de flexibilidade na configuração de tanques agitados para o processamento de diferentes tipos de produtos, realizou-se também um estudo para analisar o efeito da viscosidade do fluido no sistema de duplo impelidor Âncora - PBT. As simulações foram realizadas utilizando três viscosidades entre 25.000 e $100.00 \mathrm{cP}$. Na Tabela 3 é reportadas a potência especifica e o tempo de mistura estimado para diferentes viscosidades do fluido.

Tabela 3 - Influência da viscosidade do fluido no consumo de energia

\begin{tabular}{|c|c|c|c|}
\hline Viscosidade [cP] & $\mathrm{P} / \mathrm{V}\left[\mathrm{W} \mathrm{m}^{\wedge}-3\right]$ & $\mathrm{t}$ mistura [h] & Energia [KWh] \\
\hline 100.000 & 225,193 & 0,49 & 0,79 \\
\hline 50.000 & 28,149 & 0,98 & 0,20 \\
\hline 25.000 & 3,51864 & 1,97 & 0,05 \\
\hline
\end{tabular}

Conforme a Tabela 3 nota-se que quanto maior a viscosidade do fluido, menor é o tempo necessário na operação de mistura, porém, a potência requerida aumenta consideravelmente e, portanto é esperado um aumento no consumo de energia. Esse comportamento pode ser justificado pelo aumento da velocidade de rotação dos impelidores conforme ocorre o acréscimo na viscosidade do fluido empregado, feito para manter o valor do Reynolds desejado.

Em todos os casos, pode ser visto que, mesmo mudando a viscosidade do fluido no sistema de duplo impelidor Âncora-PBT, o tempo de homogeneização e o consumo de energia foi muito menor comparado ao sistema com impelidor Âncora. Assim, baseando-se nos resultados obtidos, o arranjo proposto Âncora-PBT aumenta a eficiência do processo de mistura no tanque.

\section{CONCLUSÃO}

Através da análise CFD pode-se concluir que a mistura no sistema Âncora-PBT é favorecida, já que o impelidor PBT ajuda na recirculação axial do fluido no tanque. Conforme os resultados, o tempo de mistura pôde ser reduzido consideravelmente com o sistema de 
impelidores PBT - Âncora obtendo reduções de até 80 vezes do tempo com fluidos altamente viscosos $\left(>0,25 \times 10^{5} \mathrm{cP}\right)$. No entanto, observa-se um aumento na potência no sistema ÂncoraPBT, que pode ser compensado devido á redução do tempo de operação, o que trará benefícios devido ao aumento de produtividade e menor gasto de energia.

Finalmente, é importante salientar que uso de CFD pode contribuir na otimização de processos visando avaliar inovações ou melhoras em equipamentos existentes que se adequem as necessidades da indústria, além disso, este trabalho permite contribuir no estado da arte em sistemas agitados com duplo impelidor, atualmente comum na indústria, porém escasso na literatura.

\section{BIBLIOGRAFIA}

BRUCATO, A.; CIOFALO, M.; GRISAFI, F.; MICALE, G. Numerical prediction of flow fields in baffled stirred vessels: A comparison of alternative modelling approaches. Chem. Eng. Science, 53, 3653-3684, 1998.

JOAQUIM JR., C.F.; CEKINSKI, E.; NUNHEZ, J. R.; URENHA, L. C. Agitação e mistura na indústria. LTC Editora, 2007.

LANE, G. L.; SCHWARZ, M. P.; EVANS, G. M. Chapter 34 - Comparison of CFD Methods for Modelling of Stirred Tanks. 10th European Conference on Mixing, (eds.) Akker, H. E. A. V. D. \& Derksen, J. J., Elsevier Science, Amsterdam, 2000.

MURTHY, B. N.; JOSHI, J. B. Assessment of standard, RSM and LES turbulence models in a baffled stirred vessel agitated by various impeller designs. Chem. Eng. Science, 63, 5468-5495, 2008.

CHHABRA, R. P.; RICHARDSON J.F. Non-Newtonian Flow: Fundamentals and Engineering Applications, 1999.

VERSTEEG, H. K.; MALALASEKERA, W. An Introduction to Computational Fluid Dynamics: The Finite Volume Method, Longman Scientific \& Technical, UK, 1995.

PAUL, E. L.; ATIEMO-OBERG, V.; KRESTA, S. M. Handbook of industrial mixing: Science and Practice. John Wiley \& Sons, 2004.

ANSYS, Inc. Solver Theory Guide. ANSYS CFX 14.0, 2011.

NUNHEZ, J. R.; KRESTA, S. M.; MACHADO, M. B.; GOMEZ, J.L. Investigating On the Influence of Mesh Density, Turbulence Models and Numerical Aspects of CFD Modeling in PBT Impellers Using Non-Structured Meshes, AIChE Annual Meeting, Pittsburgh, 526e, 282309, 2012.

CORONEO, M.; MONTANTE, G.; PAGLIANTI, A.; MAGELLI, F. CFD prediction of fluid flow and mixing in stirred tanks: Numerical issues about the RANS simulations. Comp. \& Chem. Eng., 35, 1959-1968, 2010. 